\title{
The Modification of the Responses to Noradrenaline and Acetylcholine by Aging and the Effects of Some Smooth Muscle Relaxants on the Smooth Muscle Contractile Drugs
}

\author{
Experiment in the Isolated vas Deferens of SHRSP \\ and Wistar-Kyoto Rats
}

Takao Yanagawa, Nobuyuki Yamamoto, and Aritomo Suzuki

There are many reports that the actions of the various vascular smooth muscle contractile drugs in SHRSP were reduced compared to those in normotensive rats, while there are few reports that no difference was found. It is thought that the age of rats used may be a cause of the difference observed. Thus, the authors, using the isolated vas deferens of SHRSP, investigated the responses to acetylcholine and noradrenaline by comparing to those of normotensive rats, from the standpoint of aging. In addition, the effects of some smoth muscle relaxants on the actions of contractile drugs were also investigated.

Materials and Methods:

The strips of vas deferens from SHRSP and Wistar-Kyoto rats (WKR) were hung in Locke solution according to Magnus method and the tension of the strip was isotonically recorded. The bathing solution was maintained at $28^{\circ} \mathrm{C}$ and continuously bubbled with air.

Results:

I. The modification of the contractions by noradrenaline (NA) and acetylcholine (ACh) by aging. 1) The responses to ACh and NA were compared to the maximal response to $\mathrm{K}(100 \%)$. The maximal responses to NA in SHRSP and WKR at the age of 3 months (M) were about $40 \%$ and $80 \%$ of the maximal contraction produced by $K$, respectively. The concentration-action (C-A) curve of NA in SHRSP was shifted to the right and downward compared to that of WKR. The maximal responses to NA in SHRSP and WKR at the age of $7 \mathrm{M}$ were almost the same as that of the maximal contraction by $\mathrm{K}$ and also the $\mathrm{C}-\mathrm{A}$ curve of $\mathrm{NA}$ in SHRSP was very similar to that in WKR. The maximal responses to NA in SHR$\mathrm{SP}$ and WKR at the age $11 \mathrm{M}$ were about $40 \%$ and $60 \%$, respectively. The C-A curve of NA in SHRSP was shifted to the right and downward compared to that in WKR. 2) The Maximal responses to ACh in SHRSP and WKR at the ages of 3,7 , and $11 \mathrm{M}$ were almost the same, being $60 \%$ of the maximal contraction produced by $\mathrm{K}$. The $\mathrm{C}-\mathrm{A}$ curve of $\mathrm{ACh}$ at every age tested in SHRSP was very similar to that in WKR.

II. Influences of orciprenaline, dibutyryl c. AMP, and diltiazem on the contractions by noradreanline, acetylcholine, and K. Orciprenaline showed much weak-

From the Department of Pharmacology, Kinki University School of Medicine, Osaka-fu. 
er inhibition against the contractions by NA, ACh, and $\mathrm{K}$ in SHRSP than in WKR. But dibutyryl C. AMP showed the same degree of the inhibitory effect on the contractions by the agonists in SHRSP and WKR. The inhibitory effect of diltiazem on the contraction by $K$ in SHRSP was much stronger than that in WKR.

\section{Summary with Discussion:}

The C-A curves of NA in SHRSP and WKR were modified by aging but those of ACh were not. These results suggest that the modification of receptors by aging may be produced in only adrenaline receptor and the difference observed by the investiagators on the responses to sympathomimetics may be due to the age of rats used.

It is clarified that the shift to the right of C-A curve means the decreased affinity (A) and the shift to the downward means the decreased intrinsic activity (IA). $A$ and IA in adrenaline receptor at the ages of 3 and $11 \mathrm{M}$ of SHRSP were decreased compared to those of WKR at the same ages. There are some reports that the catecholamine contents in blood of SHRSP was high at the age of 1 to $2 \mathrm{M}$. It may be thought that the increased catechomaine contents in blood influenced on adrenaline receptor and inhibited $A$ and IA, by desensitization and/or the other mechanisms. $\beta$-stimulant, oricprenaline showed weaker inhibition against the contractions by the agonists in SHRSP than those in WKR, but dibutyryl C. AMP showed almost the same degree of inhibitory effect on the contractions by the agonists in SHRSP and WKR. These results suggest that the productive activity of C. AMP by $\beta$-stimulant may be lower in SHRSP compared to that in WKR. It seems to be coincidance with the previous reports that adenylcyclase activity in SHR$\mathrm{SP}$ was lower than in normotensive rats.

$\mathrm{Ca}$ antagonist, diltiazem showed the preferential antagonism against the contraction by $\mathrm{K}$ in SHRSP. It is clarified that the $\mathrm{K}$-induced contraction is induced by only $\mathrm{Ca}$ influx, and thus it is thought that $\mathrm{Ca}$ influx may be accelerated in SHRSP. The decreased C. AMP productive activity and the increased Ca influx may be the factors of hypertension in SHRSP. 